\title{
A Differential Evolution based Optimization for Master Production Scheduling Problems
}

\author{
S. Radhika ${ }^{1}$, Ch. Srinivasa Rao ${ }^{2}$ and K. Karteeka Pavan ${ }^{3}$ \\ ${ }^{1}$ Dept. of ME, R.V.R \& J.C College of Engineering (Autonomous), Guntur \\ ${ }^{2}$ Dept. of ME, Andhra University College of Engineering (Autonomous), \\ Visakhapatnam \\ ${ }^{3}$ Dept. of IT, R.V.R. \& J.C College of Engineering (Autonomous), Guntur \\ *Corresponding Author: sajjar99@yahoo.com
}

\begin{abstract}
Heuristic evolutionary optimization algorithms are the solutions to many engineering optimization problems. Differential evolution $(D E)$ is a real stochastic evolutionary parameter optimization in current use.DE does not require more control parameters compared to other evolutionary algorithms. Master Production Scheduling (MPS) is posed as one of multi objective parameter optimization problems and often require an optimal solution for the success of a business organization by balancing demand and supply. This work reviews some of the fundamental theory of differential evolution, the methodology for master production scheduling calculation and most important results. The results available for the existing algorithms are compared with results obtained by the proposed evolutionary algorithm. The analysis reveals that the DE algorithm provides a better solution with reasonable computational time.
\end{abstract}

Keywords: Master Production Schedule, Multi-objective Optimization, Differential Evolution

\section{Introduction}

The current problem is a multi-objective nonlinear constrained optimization problem. The difficulty in optimization of engineering problems causes various optimization solutions. As a solution to these problems several heuristic algorithms have been developed for optimization of parameters. Among these one important group is evolutionary algorithms (EA) [16]. Genetic Algorithms (GA), Evolution Strategy (ES), Evolutionary Programming (EP) and Differential Evolution (DE) are some of the evolutionary algorithms. The most commonly used evolutionary optimization technique is the Genetic Algorithm (GA). Though, the GA provides a near optimal solution for complex problems, it requires a number of parameters in advance such as population size, crossover rate and mutation rate, which affect the effectiveness of the solution. Determining the optimum values for these controlling parameters is very difficult in practice [20]. Differential evolution (DE) is one of the most powerful stochastic real-parameter optimization algorithms in current use [6-8]. DE follows similar computational steps as in a standard evolutionary algorithm. Compared to other Evolutionary Algorithms DE is very simple to code. The recent studies on DE have shown that DE provides a better performance in terms of accuracy, robustness and convergence speed with its simplicity [9-11]. The number of control parameters in DE is very few compared to other algorithms [20]. 
Master production scheduling has been extensively investigated over the last three decades and it continues to attract the interest of both the academic and industrial sectors. MPS is the declaration of what the company expects to manufacture in terms of configuration, quantities and specific dates that drives Materials Requirement Planning (MRP) and other subsequent activities of a manufacturing company [1]. Master Production Schedule facilitates us to perceive what is needed, anticipating changes as well as potential shortages or surpluses that may possibly have a negative impact on any phase of an enterprise. The master plan which is often mistaken as a sales forecast, along with the pending orders, availabilities of material and capacity, managerial policies and goals turns out as one of the most important information for the system.

The MPS acts as an instrument in the hands of top management in controlling over manufacture resources and becomes the input of the downstream planning levels such as Material Requirement Planning (MRP) and Capacity Requirement Planning (CRP). Hence, inappropriate decision on the MPS development may lead to infeasible execution, which ultimately causes poor delivery performance. One must ensure that the proposed MPS is valid and realistic for implementation before it is released to real manufacturing system [2]. In practice, where production environment is stochastic in nature, the development of the MPS is no longer a simple task. The MPS creation problem becomes even more sophisticated as decision makers try to consider multi-objectives; minimizing inventory, maximizing customer satisfaction and maximizing resource utilization.

In the present work, as in most industries worldwide, the creation of an MPS considers conflicting objectives, such as maximization of service levels, efficient use of resources and minimization of inventory levels. The work presents the development and use of Differential Evolution (DE) to MPS problems, something that does not seem to have been done so far.

The present research illustrates that the use of differential evolution is a viable technique for MPS problems. However, its applicability is still heavily dependent on the size of the manufacturing scenario. As said by Higgins [3], Slack et al., [4] and Tubino [5], the most important activity in any production planning and control of production is the master production schedule (MPS). From the literature one can figure out that a company's succession and malfunction of performance are apparently based on its master plan as it coordinates market demand with the internal resources of the company. The master plan whose creation depends on the management philosophy acts as a connection between the strategic planning and production scheduling. Just in time (JIT) and theory of constraints (TOC) are the two popular philosophies usually adopted [12]. Gaither and Fraizer [13] have portrayed the master production schedule process in a JIT environment at the Japanese Toyota car manufacturer. The company which adopts the TOC philosophy, should create the MPS concentrating on the final products that use the bottle neck resources and later on their components Spencer and Cox [1].

Pertaining to these issues, several studies have suggested an authentication process to check the validity of tentative MPS. Higgins et al., [14] in order to ascertain an optimum and a realistic MPS have anticipated a simulation analysis to get executed on the tentative MPS. Kochhar et al., [15] have introduced a knowledge-based system approach, which combines human proficiency with computer computation, to attain an accurate and realistic master schedule. Heizer et al., [17] pooled a proposal about the iterative planning process that permits a planner to ensure the validity of each planning process.

In addition to the substitution of the verification process, researchers also employ various advanced optimization techniques in order to enhance MPS quality. For instance, Vieira et al., [18] applied simulated annealing to solve MPS problem and the work reveals some drawbacks of simulated annealing such as overcoming local optimum. Soares et al., [19] 
introduced new genetic algorithm structure for solving MPS problem and formulates the fitness function which aims to minimize inventory levels, maximize service level, minimize overtime and minimize inventory level below safety stock. At the end, Vieira et al., [21] has compared genetic algorithms and simulated annealing for master production scheduling problems.

The next section describes how differential evolution can be used to solve the MPS problems.

\section{Multi-objective optimization for MPS using differential evolution (MOMDE)}

This section provides a solution for MPS problem using DE by proposing a three dimensional chromosome representation, criteria for initial population and for termination.

\subsection{Chromosome representation}

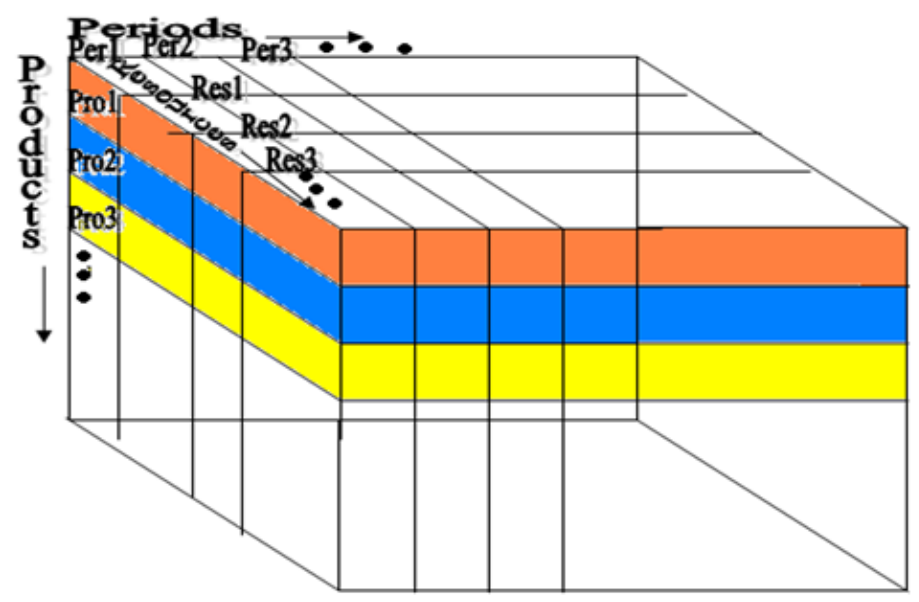

Figure 1. Structure of the Chromosome

The proposed MOMDE population contains several chromosomes. Each chromosome is in three dimensions to represent the individual solution. The conceptual model of the chromosome for the MOMDE for a scenario with products, resources, and periods is in the Figure 1. A set of genes makes a chromosome, which represents the distribution of quantities to be made at the various available resources for a given product at a specific time period. A set of chromosomes composing the chromosome group represents the total distribution of quantities to be made of all the products at every resource, in a given time period.

\subsection{Basics of differential evolution}

All evolutionary algorithms aim to improve the existing solution using the techniques of mutation, recombination and selection. The general paradigm is as follows:

2.2.1. Initialization: Creation of a population of individuals. The $i$ th individual vector (chromosome) of the population at current generation $t$ with $d$ dimensions is as follows,

$$
Z_{i}(t)=\left[Z_{i, 1}(t), Z_{i, 2}(t), \ldots, Z_{i, d}(t)\right]
$$


2.2.2. Mutation: A random change of the vector $\vec{x}$ components. It can be a single-point mutation, inversion, translocation, deletion, etc.

For each individual vector $\mathrm{Z}_{\mathrm{k}}(\mathrm{t})$ that belongs to the current population, a new individual, called the mutant individual is derived through the combination of randomly selected and prespecified individuals.

$$
\mathrm{U}_{\mathrm{k}, \mathrm{n}}(\mathrm{t})=\mathrm{Z}_{\mathrm{m}, \mathrm{n}}(\mathrm{t})+\mathrm{F} *\left(\mathrm{Z}_{\mathrm{i}, \mathrm{n}}(\mathrm{t})-\mathrm{Z}_{\mathrm{j}, \mathrm{n}}(\mathrm{t})\right)
$$

the indices $m, n, i, j$ are uniformly random integers mutually different and distinct from the current index $\mathrm{k}$, and $\mathrm{F}>0$ is a real positive parameter, called mutation or scaling factor (usually€[0,1])

2.2.3. Recombination (Crossover): merging the genetic information of two or more parent individuals for producing one or more descendants.

DE has two crossover schemes: the exponential and the binomial or uniform crossover. We have used the binomial crossover in this paper. The binomial or uniform crossover is performed on each component $n(n=1,2, \ldots, d)$ of the mutant individual $U_{k, n}(t)$. For each component a random number $r$ in the interval $[0,1]$ is drawn and compared with the crossover rate or recombination factor (another DE control parameter), $\mathrm{CR} €[0,1]$. If $\mathrm{r}<=\mathrm{CR}$, then the $\mathrm{n}^{\text {th }}$ component of the mutant individual $\mathrm{U}_{\mathrm{k}, \mathrm{n}}(\mathrm{t})$ will be selected, Otherwise, the $\mathrm{nth}$ component of the target vector $Z_{k, n}(t)$ becomes the nth component of the trial vector.

$$
U_{k}, n(t+1)=\left\{\begin{array}{l}
U k, n(t), \text { if } \operatorname{randn}(0,1)<C r \\
Z k, n(t), \text { otherwise. }
\end{array}\right.
$$

2.2.4. Selection: choice of the best individuals for the next cycle.

If the new offspring yields a better value of the objective function, it replaces its parent in the next generation; otherwise, the parent is retained in the population, i.e.,

$$
\mathrm{Z}_{\mathrm{k}}(\mathrm{t}+1)=\left\{\begin{array}{l}
\mathrm{U}_{\mathrm{k}}(\mathrm{t}+1), \text { if } \mathrm{f}\left(\mathrm{U}_{\mathrm{k}}(\mathrm{t}+1)\right)>\mathrm{f}\left(\mathrm{Z}_{\mathrm{k}}(\mathrm{t})\right) \\
\mathrm{Z}_{\mathrm{k}}(\mathrm{t}), \text { if } \mathrm{f}\left(\mathrm{U}_{\mathrm{k}}(\mathrm{t}+1)\right)<\mathrm{f}\left(\mathrm{Z}_{\mathrm{k}}(\mathrm{t})\right)
\end{array}\right.
$$

where $f(\cdot)$ is the objective function to be minimized.

\subsection{Initial population criteria}

In MOMDE the population individuals are filled up randomly, with values ranging from zero to the maximum Gross Requirement (GR) for the time period. These values always respect the standard batch (lot) size restriction (i.e., they are always multiples of the standard lot size).

\subsection{The stopping criteria}

The stopping criteria in the present work is "Stop by convergence or stagnation". The convergence of the algorithm is based on the fitness value of the fittest individual. The difference of fitness value of fittest individuals in any two successive generations is less than 0.0001 , is the stopping criteria. 


\section{The Fitness Function}

MPS problem is posed as a multi-objective optimization problem. For the optimization of the selected parameters the following multi-objective criteria is selected as the fitness function (Soares et al., 2008)

$$
\begin{gathered}
\text { fitness }=\left[\frac{1}{1+Z_{n}}\right]---(5) \\
\text { Where } Z_{n}=c_{1} \frac{A I L}{A I L_{\max }}+c_{2} \frac{R N M}{R N M_{\max }}+c_{3} \frac{B S S}{B S S_{\max }}+c_{4} O C--(6)
\end{gathered}
$$

$\mathrm{EI}_{\max }, \mathrm{RNM}_{\max }$, and $\mathrm{BSS}_{\max }$, are the biggest values found from the initial population created. Unit values are used for the fitness coefficients $c_{1}, c_{2}, c_{3}$ and $c_{4}-$ which indicate equal importance among the objectives to be minimized. Master Production schedule problem can be mathematically modeled as a mixed integer problem as follows [19]:

$$
\text { Minimize: } Z=c_{1} A I L+c_{2} R N M+c_{3} B S S+c_{4} O C---(7)
$$

The constraints and the nomenclature used are taken from [19]. The subsequent section demonstrates the applicability of DE in finding a master schedule plan for a production scenario.

\section{MPS problem considered}

A manufacturing scenario is selected from Soares et al., [19] to study the applicability of Differential Evolution algorithm for the MPS problem as follows.

The scenario is with a planning horizon of 13 periods, four productive resources, and 20 different products. The scenario also considered (a) different period lengths (b) different initial inventory quantity for each product and (c) different safety inventory levels and different standard production lot sizes

\section{Results and discussion}

The applicability of the proposed MOMDE was tested on the manufacturing scenario considered. The plot on figure 2 shows the variations of fitness evolution in all the 50 independent runs. The best fitness value 0.867919601 is obtained in the $32^{\text {nd }}$ run and the worst fitness value 0.866834744 is obtained in the $46^{\text {th }}$ run. The fitness is increased by nearly $20 \%$ to that when done with GA and the average number of iterations taken for the convergence is 4 . 


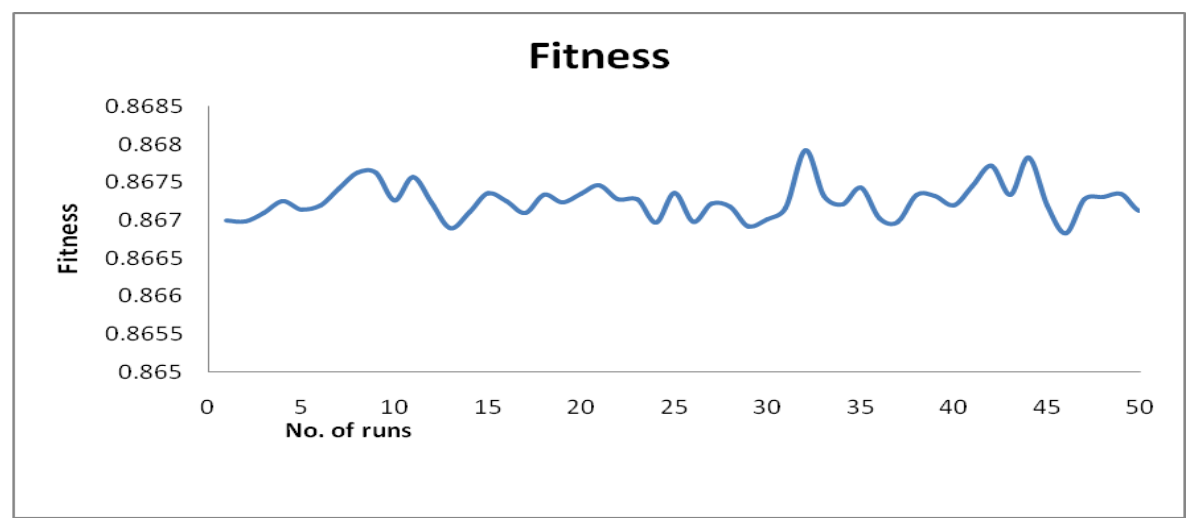

Figure 2. Evolution of fitness values

The best master production schedule found with respect to the 4 resources, 13 periods for all the 20 products along with the total MPS for each product is shown in the Table 1.

Table 1. Best mps obtained

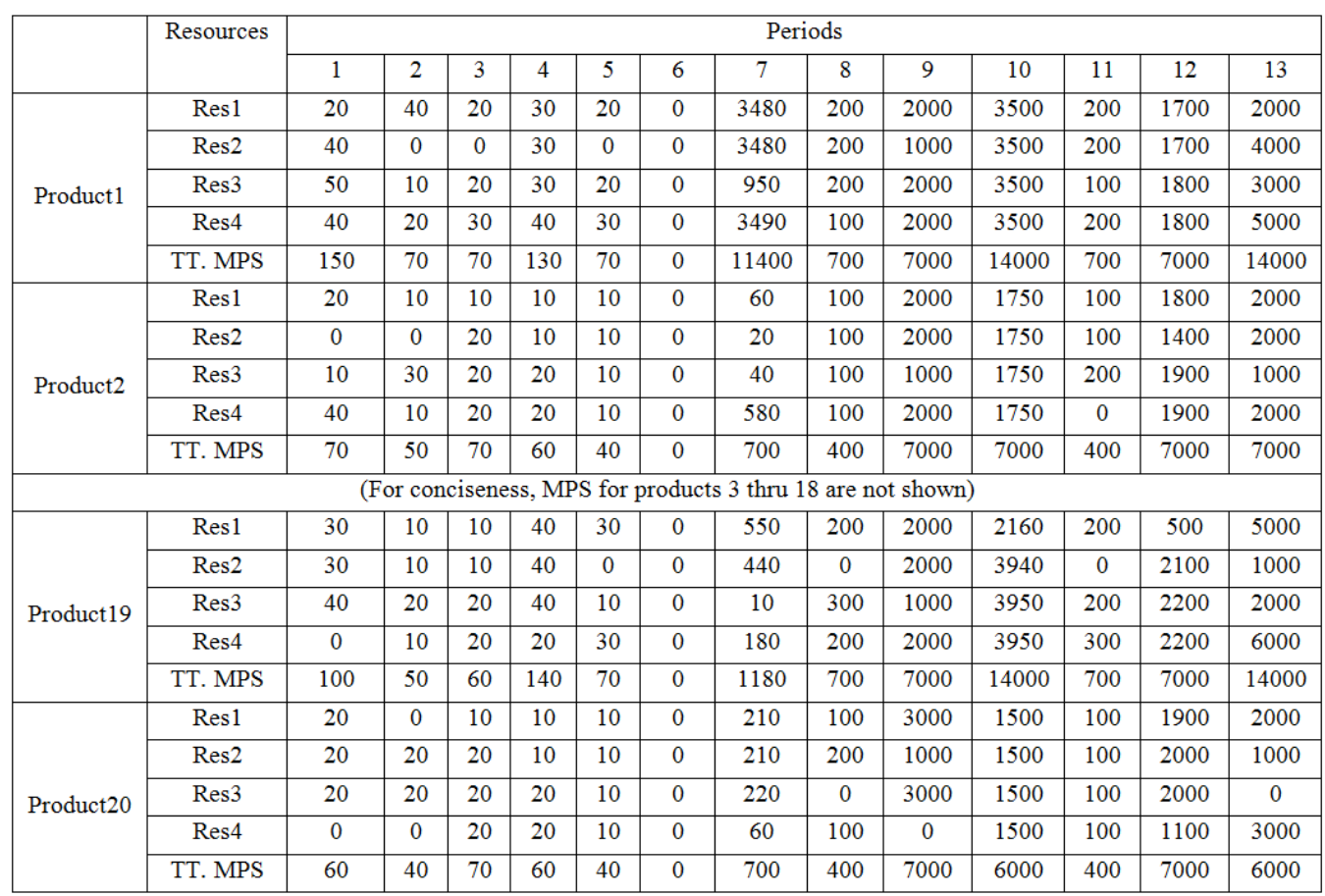

Figure 3 shows the evolution of the normalized performance measures considered in the multi objective fitness function which include: average ending inventory (EI), requirements not met (RNM), and inventory below safety stock (BSS). 


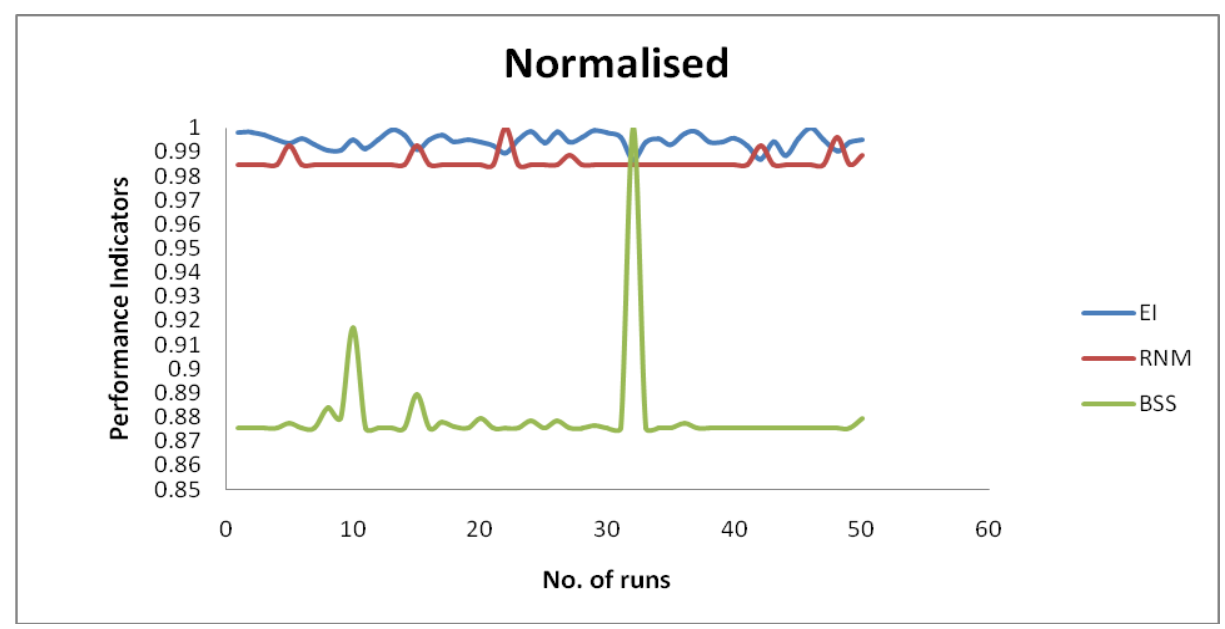

Figure 3. Normalized performance indicators in terms of number of runs

This work showed that master plan created with MOMDE presented low levels of ending inventory, low levels of requirements not met and efficiently met safety inventory levels. Also, the results show that the DE approach gives a better result when compared to the existing work with GE. Table 2 shows the comparison of the various parameters obtained through MOMDE with those of MPS GA [19].

Table 2. Comparison between the values of performance indicators

\begin{tabular}{|c|c|c|}
\hline & GA & DE \\
\hline Fitness & 0.6679 & 0.867259 \\
\hline EI (units/hour) & 4555.08 & 5363.246 \\
\hline RNM (units/hour) & 321.42 & 200.2308 \\
\hline BSS (units/hour) & 37.03 & 12.53789 \\
\hline
\end{tabular}

\section{Conclusions and Future Scope}

The complexity of parameter optimization problems increases with the increase in the number of parameters. The present MOMDE model is useful for future research, to generate a more advanced model for improved reliability in MPS. The results demonstrate that the MOMDE produce more optimal values compared to GA. Application of the proposed MOMDE in a larger production scenario and testing its validity to an industry will be our upcoming work. Also Incorporation of self adaptation of parameters for DE for improved reliability is our future endeavor. Defining a more suitable fitness function by considering different weights to the coefficients and their influence may be analyzed.

\section{References}

[1] M. S. Spenser and J. F. Cox III, "Master production scheduling development in a theory of constraints environment", Prod Inv Manage J., vol. 1, no. 36, (1995), pp. 8-14.

[2] I. Supriyanto, "Fuzzy Multi-Objective Linear Programming and Simulation Approach to the Development of Valid and Realistic Master Production Schedule”, LJ_proc_supriyanto_de 201108_01, (2011). 
[3] P. Higgins and J. Browne, "Master production scheduling: a concurrent planning approach", Prod Plan Control, vol. 3, no. 1, (1992), pp. 2-18.

[4] N. Slack, S. Chambers and R. Johnston, "Operations management", 4th Ed., (2004).

[5] D. F. Tubino, "Manual do planejamento e controle daprodução", 2nd edn., (2000).

[6] K. V. Price, "Differential evolution vs. the functions of the 2nd ICEO", Proc. IEEE Int. Conf. Evol. Comput., (1997), pp. 153-157.

[7] K. V. Price and R. Storn, "Differential evolution: A simple evolution strategy for fast optimization", Dr. Dobb's J., vol. 22, no. 4, (1997), pp. 18-24.

[8] R. Storn and K. V. Price, "Differential evolution: A simple and efficient adaptive scheme for global optimization over continuous spaces", ICSI, USA, Tech. Rep. TR-95-012, (1995).

[9] R. Storn and K. V. Price, "Minimizing the real functions of the ICEC 1996 contest by differential evolution", Proc. IEEE Int. Conf. Evol.Comput., (1996), pp. 842-844.

[10] R. Storn, "On the usage of differential evolution for function optimization", Proc. North Am. Fuzzy Inform. Process. Soc., (1996), pp. 519-523.

[11] R. Storn and K. Price, "Differential evolution: A simple and efficient heuristic for global optimization over continuous spaces", J. Global Optimization, vol. 11, no. 4, (1997), pp. 341-359.

[12] M. L. Sale and A. R. Inman, "Survey-based comparison and change in performance of firms using traditional manufacturing”, JIT and TOC Int J Prod Res., vol. 41, no. 04, (2003), pp. 829-844.

[13] N. Gaither and G. Frazier, "Administração da produção e operações”, 8th edn. Pioneira Thomson Learning, São Paulo, (2000).

[14] P. Higgins and J. Browne, "Master production scheduling: a concurrent planning approach", Prod Plan Control, vol. 3, no. 1, (1992), pp. 2-18.

[15] A. K. Kochhar, X. Ma and M. N. Khan, "Knowledge-based systems approach to the development of accurate and realistic master production schedules", Journal of Engineering Manufacture, vol. 212, (1998), pp. 453-60.

[16] S. Das, A. Abraham and A. Konar, "Metaheuristic Clustering", SCI 178, Springer-Verlag Berlin Heidelberg, (2009), pp. 63-110.

[17] J. H. Heizer and B. Render, “Operations Management”, Upper Saddle River, New York: Pearson Prentice Hall, (2006).

[18] G. E. Vieira and C. P. Ribas, "A new multi-objective optimization method for master production scheduling problems using simulated annealing", International Journal of Production Research, vol. 42, (2004).

[19] M. M. Soares and G. E. Vieira, "A New multi-objective optimization method for master production scheduling problems based on genetic algorithm", International Journal of Advanced Manufacturing Technology, vol. 41, (2009), pp. 549-567.

[20] S. Das, P. N. Suganthan, "Differential Evolution: A Survey of the State-of-the-Art", IEEE Transactions On Evolutionary Computation, vol. 15, no. 1, (2011), pp. 4-32.

[21] G. E. Vieira, F. Favaretto and P. C. Ribas, "Comparing genetic algorithms and simulated annealing in master production scheduling problems", Proceeding of 17th International Conference on Production Research (2003) Blacksburg, Virginia, USA. 